\title{
Stenotrophomonas maltophilia endophthalmitis following cataract surgery: clinical and microbiological results
}

This article was published in the following Dove Press journal:

Clinical Ophthalmology

18 April 2013

Number of times this article has been viewed

\section{Jonathan S Chang \\ Harry W Flynn Jr \\ Darlene Miller \\ William E Smiddy}

Department of Ophthalmology, Bascom Palmer Eye Institute, Miller School of Medicine, University of Miami, Miami, FL, USA
Correspondence: Jonathan S Chang Bascom Palmer Eye Institute, 900 NW 17th Street, Miami, FL 33।36, USA

$\mathrm{Tel}+\mathrm{I} 3053266000$

Fax +I 3053266417

Email jchang7@med.miami.edu
Background: Stenotrophomonas maltophilia is a Gram-negative organism known to cause opportunistic infections. It is a rare source of endophthalmitis, often in the setting of trauma, but has been reported following cataract extraction. The purpose of this study was to evaluate antimicrobial sensitivities, clinical characteristics, and treatment outcomes in patients with endophthalmitis caused by S. maltophilia following cataract extraction.

Methods: A retrospective case review of records from January 1, 1990 to June 30, 2010 was performed at the Bascom Palmer Eye Institute.

Results: Eight cases of S. maltophilia endophthalmitis were identified following cataract surgery. Initial visual acuity ranged from 20/200 to light perception. Time to diagnosis with cultures was 2-118 days. Patients received either intravitreal tap and inject $(n=5)$ or pars plana vitrectomy with intravitreal antibiotic injections $(n=3)$. All patients had vitreous or anterior chamber cultures positive for $S$. maltophilia. Seven of seven isolates tested were found to be sensitive to ceftazidime. Seven of eight isolates were sensitive to polymyxin B, six of eight isolates were sensitive to amikacin, and five of the seven isolates tested were sensitive to ciprofloxacin. Two of four tested isolates were sensitive to trimethoprim-sulbactam. All eight isolates were resistant to gentamicin and seven of the seven tested isolates were resistant to imipenem. All patients received intravitreal ceftazidime as part of the initial treatment regimen. Final visual acuity ranged from $20 / 25$ to $4 / 200$.

Conclusion: S. maltophilia endophthalmitis is a rare source of endophthalmitis following cataract surgery. A case series of eight independent patients is reported, along with antibiotic resistance profiles and clinical outcomes. Isolates showed sensitivity to ceftazidime, amikacin, and polymyxin, with variable sensitivity to other antibiotics, therefore differing from previous reports.

Keywords: endophthalmitis, Stenotrophomonas maltophilia, cataract

\section{Introduction}

Stenotrophomonas maltophilia is a Gram-negative bacillus that causes many opportunistic infections. ${ }^{1}$ Previously identified as Pseudomonas maltophilia or Xanthomonas maltophilia, it has been reported to cause sepsis, pneumonia, urinary tract infection, meningitis, endocarditis, septic arthritis, and peritonitis. ${ }^{1}$ It has also been noted to be a pathogen in many ocular infections, including conjunctivitis, keratitis, dacryocystitis, cellulitis, infected scleral buckles, and endophthalmitis. ${ }^{2-16}$ As a cause of endophthalmitis, the mechanism has been reported as endogenous, ${ }^{17}$ or exogenous, such as posttrauma, ${ }^{18-21}$ contamination of irrigation fluid, ${ }^{22,23}$ and following intraocular surgery. ${ }^{24-31}$ Presentation may be acute or subacute, with varying degrees of inflammation. This study reports a retrospective case series of eight independent cases of endophthalmitis 
after cataract surgery caused by $S$. maltophilia treated at the Bascom Palmer Eye Institute (BPEI) in Miami, FL, USA.

\section{Materials and methods}

The study was approved by the University of Miami Miller School of Medicine institutional review board. The study design was a retrospective case review of records. Using a database of microbiological isolates from the Department of Microbiology at BPEI, vitreous and anterior chamber cultures positive for S. maltophilia were identified between January 1, 1990 and June 30, 2010. At the time of culture, an automated Vitek 2 system (bioMérieux Inc, Hazelwood, MO, USA) was used for identification and sensitivities. Supplementary tests were performed with Etests and disk diffusion, as indicated by the Clinical Laboratory Standards Institute. Clinical records were then reviewed for clinical presentation, treatment, and outcomes. Inclusion criteria were prior cataract surgery and single organism on culture. Of 1345 positive vitreous cultures, nine cases were identified as S. maltophilia. Eight of these cases were following cataract surgery and one case was excluded because of the presence of multiple organisms. One case was identified from a positive anterior chamber culture. Four of the cases (cases 1-4) had been reported earlier in the literature under the previous name of $X$. maltophilia. ${ }^{26}$ The original clinical records of these patients were reviewed independently for this study.

\section{Results}

The characteristics of the eight cases are summarized in Table 1 . The median age of patients was 75 (range 46-81) years.
Five of the cases were referred from ophthalmologists in the Miami metropolitan area, two were from international locations (Venezuela and Nicaragua), and one case was being monitored after lens repositioning at BPEI. Patients presented with decreased vision and pain. The initial visual acuity varied from 20/200 to light perception. Seven of the cases presented within two to 35 days after surgery. One case (case 2) presented 118 days after surgery and had been treated by an outside provider prior to being seen at BPEI. Three of the patients were treated with pars plana vitrectomy and intravitreal injection of antibiotics, and five initially received vitreous tap and intravitreal injection of antibiotics. Six patients received dexamethasone as part of their initial treatment regimen along with antibiotics. Two patients (cases 1 and 4) initially improved, but subsequently worsened following initial management, requiring further intervention. Final visual acuity outcomes ranged from 20/20 to $4 / 200$. One patient had the intraocular lens removed. There were no retinal detachments or suprachoroidal hemorrhages. Antibiotic resistance profiles are detailed in Table 2. Seven of eight isolates were tested for and demonstrated sensitivity to ceftazidime. Six of eight isolates showed sensitivity to polymyxin B. Resistance to gentamicin was noted in all eight isolates. Resistance to imipenem was noted in seven of seven tested isolates.

\section{Case report I}

A 48-year-old man underwent extracapsular cataract extraction in Nicaragua. On the first postoperative day, visual acuity was reported as 20/20. He developed pain, elevated intraocular

Table I Demographics and clinical features of cases of endophthalmitis caused by Stenotrophomonas maltophilia

\begin{tabular}{|c|c|c|c|c|c|c|c|c|c|}
\hline $\begin{array}{l}\text { Case } \\
\text { (eye, age) }\end{array}$ & Year & $\begin{array}{l}\text { Surgery } \\
\text { (location) }\end{array}$ & $\begin{array}{l}\text { Postoperative } \\
\text { days to evaluation }\end{array}$ & $\begin{array}{l}\text { Initial } \\
\text { VA }\end{array}$ & $\begin{array}{l}\text { Initial } \\
\text { treatment }\end{array}$ & Antibiotics & Steroid & Recurrence & $\begin{array}{l}\text { Final } \\
\text { VA }\end{array}$ \\
\hline I $(\mathrm{OD}, 47)$ & 1996 & ECCE (Nicaragua) & 5 & LP & $\begin{array}{l}\text { PPV, } \\
\text { IV antibiotics }\end{array}$ & $\begin{array}{l}\text { Vancomycin, } \\
\text { ceftazidime }\end{array}$ & + & + & $4 / 200$ \\
\hline $2(\mathrm{OD}, 46)$ & 1996 & $\begin{array}{l}\text { Phaco/IOL } \\
\text { (Venezuela) }\end{array}$ & 118 & $20 / 200$ & $\begin{array}{l}\text { PPV, } \\
\text { IV antibiotics }\end{array}$ & Ceftazidime & - & - & $20 / 30$ \\
\hline $3(\mathrm{OS}, 69)$ & 1999 & Phaco/IOL (Miami) & 35 & HM & Tap and inject & $\begin{array}{l}\text { Vancomycin, } \\
\text { ceftazidime }\end{array}$ & - & - & $20 / 20$ \\
\hline $4(\mathrm{OS}, 80)$ & 1999 & Phaco/IOL (Miami) & 17 & HM & Tap and inject & $\begin{array}{l}\text { Vancomycin, } \\
\text { Ceftazidime }\end{array}$ & + & + & $20 / 30$ \\
\hline $5(\mathrm{OD}, 8 \mathrm{I})$ & 2002 & $\begin{array}{l}\text { Phaco/IOL, lens } \\
\text { repositioning (Miami) }\end{array}$ & 2 & HM & Tap and inject & $\begin{array}{l}\text { Vancomycin, } \\
\text { ceftazidime }\end{array}$ & + & - & $20 / 150$ \\
\hline $6(\mathrm{OD}, 74)$ & 2004 & Phaco/IOL (Miami) & 22 & $\mathrm{CF}$ & Tap and inject & $\begin{array}{l}\text { Vancomycin, } \\
\text { ceftazidime }\end{array}$ & + & - & $20 / 300$ \\
\hline $7(\mathrm{OD}, 76)$ & 2005 & Phaco/IOL (Miami) & 10 & $20 / 200$ & Tap and inject & $\begin{array}{l}\text { Vancomycin, } \\
\text { ceftazidime }\end{array}$ & + & - & $20 / 70$ \\
\hline $8(\mathrm{OS}, 79)$ & 2005 & Phaco/IOL (Miami) & 16 & LP & $\begin{array}{l}\text { PPV, } \\
\text { IV antibiotics }\end{array}$ & $\begin{array}{l}\text { Vancomycin, } \\
\text { ceftazidime }\end{array}$ & + & - & $20 / 50$ \\
\hline
\end{tabular}

Abbreviations: -, negative; +, positive; ECCE, extracapsular cataract extraction; Phaco, phacoemulsification; IOL, intraocular lens implantation; LP, light perception; $\mathrm{HM}$, hand motion; CF, count fingers; PPV, pars plana vitrectomy; IV, intravitreal; VA, visual acuity. 
Table 2 Antibiotic resistance profile for cases of endophthalmitis caused by Stenotrophomonas maltophilia

\begin{tabular}{lllllllll}
\hline & \multicolumn{2}{l}{ Case } & & & & & & \\
\cline { 2 - 9 } & $\mathrm{I}$ & $\mathbf{2}$ & $\mathbf{3}$ & $\mathbf{4}$ & $\mathbf{5}$ & $\mathbf{6}$ & $\mathbf{7}$ & $\mathbf{8}$ \\
\hline Amikacin & $\mathrm{R}$ & $\mathrm{R}$ & $\mathrm{S}$ & $\mathrm{S}$ & $\mathrm{R}$ & $\mathrm{S}$ & $\mathrm{S}$ & $\mathrm{S}$ \\
Ceftazidime & $\mathrm{S}$ & $\mathrm{S}$ & $\mathrm{S}$ & $\mathrm{S}$ & $\mathrm{S}$ & $\mathrm{S}$ & $\mathrm{S}$ & $\mathrm{N} / \mathrm{A}$ \\
Ciprofloxacin & $\mathrm{S}$ & $\mathrm{R}$ & $\mathrm{S}$ & $\mathrm{S}$ & $\mathrm{R}$ & $\mathrm{S}$ & $\mathrm{S}$ & $\mathrm{N} / \mathrm{A}$ \\
Levofloxacin & $\mathrm{N} / \mathrm{A}$ & $\mathrm{N} / \mathrm{A}$ & $\mathrm{N} / \mathrm{A}$ & $\mathrm{N} / \mathrm{A}$ & $\mathrm{N} / \mathrm{A}$ & $\mathrm{S}$ & $\mathrm{S}$ & $\mathrm{S}$ \\
Gentamicin & $\mathrm{R}$ & $\mathrm{R}$ & $\mathrm{R}$ & $\mathrm{R}$ & $\mathrm{R}$ & $\mathrm{R}$ & $\mathrm{R}$ & $\mathrm{R}$ \\
Tobramycin & $\mathrm{R}$ & $\mathrm{R}$ & $\mathrm{S}$ & $\mathrm{S}$ & $\mathrm{R}$ & $\mathrm{S}$ & $\mathrm{S}$ & $\mathrm{S}$ \\
Imipenem & $\mathrm{R}$ & $\mathrm{R}$ & $\mathrm{R}$ & $\mathrm{R}$ & $\mathrm{R}$ & $\mathrm{R}$ & $\mathrm{R}$ & $\mathrm{N} / \mathrm{A}$ \\
Trimethoprim- & $\mathrm{R}$ & $\mathrm{I}$ & $\mathrm{S}$ & $\mathrm{S}$ & $\mathrm{N} / \mathrm{A}$ & $\mathrm{N} / \mathrm{A}$ & $\mathrm{N} / \mathrm{A}$ & $\mathrm{R}$ \\
sulfamethoxazole & & & & & & & & \\
Polymyxin B & $\mathrm{I}$ & $\mathrm{S}$ & $\mathrm{S}$ & $\mathrm{S}$ & $\mathrm{S}$ & $\mathrm{S}$ & $\mathrm{S}$ & $\mathrm{S}$ \\
\hline
\end{tabular}

Abbreviations: S, sensitive; R, resistant; I, intermediate; N/A, not available.

pressure, and blurred vision on the third postoperative day. On the fifth postoperative day, visual acuity was reported as no light perception, with a $20 \%$ hypopyon and anterior chamber membranes. The patient was referred to BPEI and was seen on the eighth postoperative day. Examination at that time revealed light perception vision, with intraocular pressure $27 \mathrm{mmHg}$, corneal edema and haze, 4+ anterior chamber cells, $20 \%$ hypopyon, and a pupillary membrane.

The patient was diagnosed with endophthalmitis and underwent pars plana vitrectomy with intravitreal vancomycin, ceftazidime, and dexamethasone injections. Vitreous cultures were positive for $S$. maltophilia. One week after surgery, the patient returned with increasing pain, and a second vitreous culture and repeat intravitreal injection of ceftazidime were performed. A second vitreous culture was again positive for $S$. maltophilia sensitive to ceftazidime. Five days later, the patient had increased pain with a stable clinical examination, and a repeat intravitreal injection of ceftazidime was given, along with intravitreal dexamethasone. Three weeks later, the patient continued to have pain and had another pars plana vitrectomy for vitreous opacities. Two months after surgery, the final visual acuity was $4 / 200$, and chronic fibrin was noted in the anterior chamber. The patient returned to Nicaragua and was lost to follow-up.

\section{Case report 2}

A 40-year-old man underwent phacoemulsification with intraocular lens implantation in his native Venezuela. He developed worsening vision and was treated in Venezuela with intravitreal tap and injection of amikacin and cefazolin. The vitreous tap demonstrated Gram-negative rods and he was diagnosed with presumptive Escherichia coli endophthalmitis. These cultures were not re-evaluated at BPEI. Because of chronic inflammation, the patient was
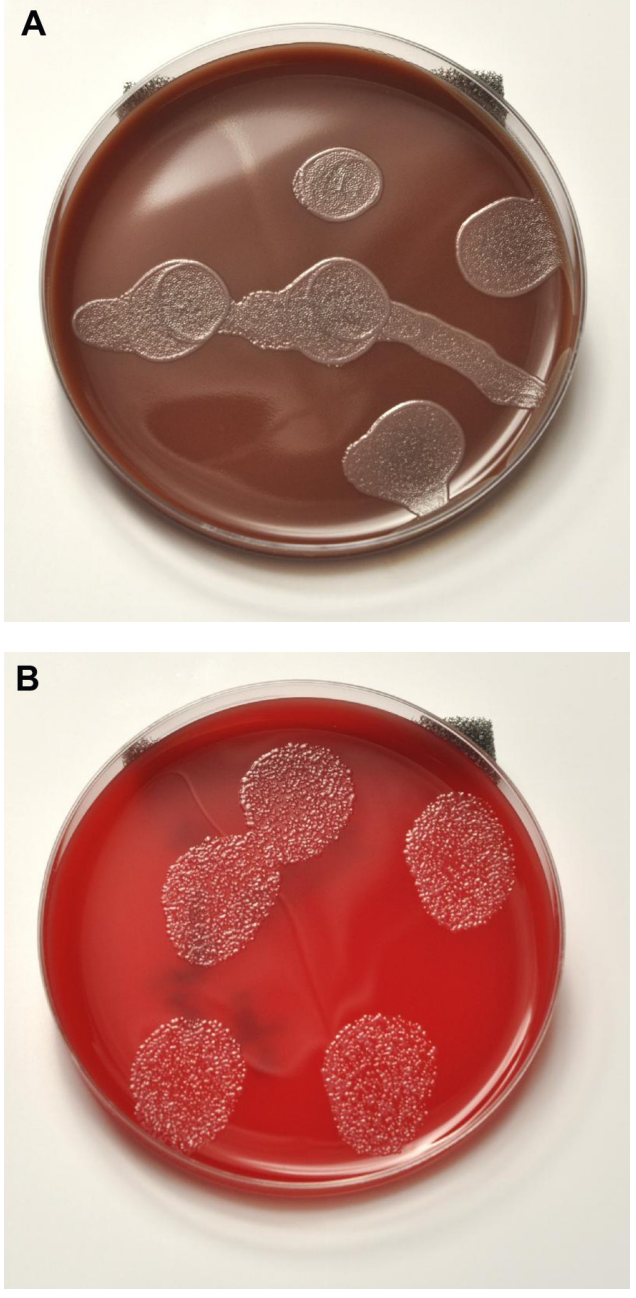

Figure I (A) Chocolate agar plate with growth of Stenotrophomonas maltophilia (B) Blood agar plate with growth of Stenotrophomonas maltophilia.

referred to BPEI and seen 118 days following the initial surgery. Examination at that time demonstrated visual acuity of $20 / 200$ in the operated eye. The cornea was clear, with $3+$ cells in the anterior chamber. No hypopyon was noted. An intraocular lens with iridocapsular adhesions was noted. On dilated fundus examination, optic nerve hyperemia, cystoid macular edema, and layered vitreous hemorrhage were noted. Retained lens fragments and cortical material were not observed. The patient was diagnosed with chronic endophthalmitis and underwent pars plana vitrectomy with intravitreal ceftazidime. He was given oral ciprofloxacin. Vitreous cultures were positive for S. maltophilia sensitive to ceftazidime and resistant to amikacin. Seven months after surgery, visual acuity was measured as 20/30.

\section{Case report 3}

A 69-year-old man underwent phacoemulsification and intraocular lens implantation by an outside ophthalmologist. 
On the first postoperative visit, visual acuity was measured as 20/25. A fragment of lens cortex was noted in the anterior chamber with trace cells and flare. The patient received a sub-Tenon's injection of triamcinolone. One week later, he was referred to BPEI. At the time of referral, visual acuity was hand motions with 1-2+ corneal folds, 3-4+ anterior chamber cells, 5\% hypopyon, and dense vitreous opacities and membranes on ultrasound. A vitreous tap and intravitreal injection of vancomycin, ceftazidime, and dexamethasone was performed. He received one dose of intravenous ceftazidime and oral chloramphenicol. Vitreous cultures were positive for $S$. maltophilia. The patient had a final recorded visual acuity of 20/20.

\section{Case report 4}

An 80-year-old woman underwent phacoemulsification and intraocular lens implantation by an outside ophthalmologist. One week following surgery, she was noted to have visual acuity of 20/25. She was referred to BPEI 17 days following surgery with visual acuity hand motions, 1+ corneal edema, $2+$ anterior chamber flare, and $0.5 \mathrm{~mm}$ hypopyon. There was no view of the posterior pole because of dense vitreous opacities. A vitreous tap and intravitreal injection of vancomycin, ceftazidime, and dexamethasone was performed. Three weeks following injection, visual acuity was 20/100 with decreased inflammation. Vitreous cultures demonstrated $S$. maltophilia. The patient returned two weeks later with visual acuity of light perception and was diagnosed with recurrent endophthalmitis. Sensitivities from the original vitreous sample demonstrated resistance to ceftazidime, and a second tap and injection of amikacin was performed. Vitreous cultures from the second sample demonstrated S. maltophilia with intermediate sensitivity to ceftazidime and sensitivity to amikacin. Two days following this second intervention, the patient's inflammation worsened, and a pars plana vitrectomy was performed with intravitreal injection of amikacin. Vitreous cultures from the vitrectomy demonstrated S. maltophilia sensitive to ceftazidime and amikacin. During the pars plana vitrectomy, the patient's silicone intraocular lens was placed in the anterior chamber because of a posterior capsular rupture. A lens exchange was performed three years later, and at the patient's final follow-up, best corrected visual acuity was 20/30.

\section{Case report 5}

An 81-year-old man underwent phacoemulsification surgery with implantation of a multifocal intraocular lens. At the time of surgery, the posterior lens capsule was ruptured and the lens was displaced. Visual acuity was measured as $20 / 25$. Two years after the initial surgery, the intraocular lens was repositioned. The irrigation fluid from the procedure contained gentamicin and vancomycin. Nine days after lens repositioning, the patient presented with hand motion vision. A vitreous tap and intravitreal injection of vancomycin, ceftazidime, and dexamethasone was performed. The following day, the patient's symptoms and clinical examination had not improved significantly. Because the Gram stain demonstrated Gram-negative rods, the patient was reinjected with ceftazidime. Vitreous cultures were positive for $S$. maltophilia sensitive to ceftazidime and resistant to amikacin. The patient returned to Long Island, NY, and the last reported follow-up was visual acuity of 20/150 one month after surgery.

\section{Case report 6}

A 74-year-old man had phacoemulsification and intraocular lens implantation in the right eye by an outside ophthalmologist. On presentation to BPEI at 26 days after surgery, visual acuity was counting fingers, with corneal edema and folds. The anterior chamber demonstrated $2-3+$ cells with hypopyon. Vitreous tap and intravitreal injection of vancomycin and ceftazidime was performed. Vitreous cultures were positive for $S$. maltophilia sensitive to ceftazidime and amikacin. At the last recorded follow-up visit, one month after tap and inject, visual acuity was 20/300.

\section{Case report 7}

A 75-year-old man underwent phacoemulsification and intraocular lens implantation by an outside ophthalmologist. He was referred to BPEI 9 days after surgery, with visual acuity 20/200, corneal edema, and anterior chamber fibrin and hypopyon. The patient was treated with vitreous tap and intravitreal injection of vancomycin, ceftazidime, and dexamethasone. Cultures were positive for S. maltophilia sensitive to ceftazidime and amikacin. One month following treatment, visual acuity was 20/70.

\section{Case report 8}

A 79-year-old woman presented to BPEI 16 days after phacoemulsification and intraocular lens implantation by an outside ophthalmologist. Visual acuity was light perception at the time of presentation. Examination demonstrated corneal edema and folds, 2+ hypopyon with 4+ anterior chamber cells, and no view of the retina. The patient underwent pars plana vitrectomy with intravitreal injections of vancomycin, ceftazidime, and dexamethasone. The patient was also treated with oral gatifloxacin. Purulent material from the anterior 
chamber was sent for culture and found to be positive for S. maltophilia. One week after surgery, the patient was noted to have increased hypopyon on examination. A repeat anterior chamber culture was performed with no growth. The patient was seen in follow-up for 1.5 months and had final visual acuity of $20 / 50$.

\section{Discussion}

S. maltophilia is a rare source of ocular infection. The first reported case of $S$. maltophilia endophthalmitis was in 1989 after implantation of a ganciclovir implant in a patient with acquired immune deficiency syndrome. ${ }^{24}$ This patient eventually required pars plana vitrectomy and removal of the implant, along with intravitreal and systemic antibiotic therapy. The overall frequency of $S$. maltophilia as a causative organism for endophthalmitis is not well documented. In the Endophthalmitis Vitrectomy Study, Gram-negative organisms accounted for $6.1 \%$ of cases. ${ }^{32}$ In 2008, Lalwani et al reported $9.6 \%$ cases of endophthalmitis in a review of 73 post-surgical endophthalmitis cases treated at BPEI. ${ }^{33}$

Horster et al reported an outbreak of $S$. maltophilia endophthalmitis in a series of 26 patients following cataract surgery. ${ }^{22}$ In this series, all patients had surgery within two days at the same hospital. The irrigation solution was found to be the source of the pathogen. Favorable visual outcomes were achieved, and all patients were treated promptly with intravitreal antibiotics and pars plana vitrectomy. The strains were confirmed by restriction fragment length polymorphism and pulsed field gel electrophoresis typing. This rapid diagnosis and treatment differs from our cases, where six of our eight cases presented after ten postoperative days or more. In cases 1 and 5, the inflammatory response and infection occurred within one week. Cases 4, 6, and 7 occurred within approximately 1-3 weeks after surgery, and cases 2 and 3 were reported more than one month after surgery. In case 2, more than 100 days had elapsed before definitive therapy was given. This suggests that, in some cases, the organism is less virulent than other organisms implicated in endophthalmitis, and may be responsible for acute or chronic endophthalmitis. No other unique clinical features were identified in this case review. Other reports of postoperative $S$. maltophilia endophthalmitis have contained few patients or multiple organisms causing endophthalmitis. ${ }^{30}$

In this study, seven of the seven tested isolates were noted to be sensitive to ceftazidime. Case 8 , who did not have ceftazidime sensitivity data, was treated with ceftazidime and improved, although this patient was also treated with oral gatifloxacin. A previous report of endophthalmitis by
Chen et al demonstrated a high frequency of $S$. maltophilia resistant to ceftazidime. ${ }^{31}$ Penland et al reported antimicrobial susceptibilities of 15 ocular isolates of $S$. maltophilia, and found resistance to ceftazidime in five of 15 isolates. ${ }^{2}$ The reason for this difference in sensitivities may be related to selective pressure of antibiotic use in differing geographic regions or different time periods of study. Also, the methodology, resistance criteria, and media used for antibiotic sensitivities may have differed. Ceftazidime is often a first-line intravitreal antibiotic agent, chosen because of its wide coverage and low intraocular toxicity. Another common first-line option is amikacin, although fewer isolates (six of eight) were sensitive to amikacin than to ceftazidime in our series.

The series presented in this paper includes seven of eight isolates sensitive to polymyxin B. Two of five tested isolates were sensitive to trimethoprim-sulfamethoxazole, a therapeutic agent commonly used for $S$. maltophilia. Three isolates were not tested, and two demonstrated mixed sensitivity or resistance to trimethoprim-sulfamethoxazole. Five of the seven isolates tested were sensitive to ciprofloxacin, and three of three tested isolates were sensitive to levofloxacin. Fluoroquinolones are another common antibiotic class used in the treatment of $S$. maltophilia. Susceptibility to tetracyclines was not investigated. Penland et al noted high susceptibility rates to the aminoglycosides (gentamicin and tobramycin), with 12 of 15 isolates sensitive to both agents. ${ }^{2}$ In our series, none of the eight isolates were sensitive to gentamicin, and five of eight were sensitive to tobramycin.

Inflammation may also play a role in the vision loss as well. Six of the eight patients in this series were treated with intravitreal steroids. The two patients who did not receive intravitreal steroids had the best visual acuity of the group, but the numbers were too low and too many variables were involved in treatment to make any meaningful comparison. The two patients who were not treated with dexamethasone also had the longest time to presentation, which may indicate they had less virulent infection.

Limitations of this study include those of any retrospective review. Because postoperative endophthalmitis has become increasingly rare, and the organism in question is atypical, the number of cases available was small. Antibiotic susceptibilities were measured ex vivo, and may not completely represent the complete in vivo state. In the case of polymyxin B susceptibility testing, there may have been some variation between the disk diffusion and minimum inhibitory concentration methods.

In case 4 , three separate vitreous cultures were performed, with differing sensitivity results for ceftazidime. 
The first culture showed resistance to ceftazidime, the second had intermediate sensitivity, and the third was sensitive. One possibility is that the resistance testing may have had some variability. It is also possible that there was selective pressure after initial injection of antibiotics, causing a change in the resistance profile for the organism.

$S$. maltophilia is a rare causative organism for endophthalmitis. This study represents a review of several cases treated at an academic referral center following cataract surgery. Visual outcomes demonstrated visual acuity better than 20/150 in the majority of cases. Antibiotic resistance testing showed sensitivity to ceftazidime and amikacin, suggesting that these antibiotics may be useful as intravitreal therapy in the treatment and prevention of this pathogen. Sensitivity to polymyxin B suggests that this agent may be a useful topical antibiotic for prevention.

\section{Acknowledgment}

This work was supported in part by Research to Prevent Blindness, New York, NY.

\section{Disclosure}

HWF is a consultant to Alimira, Pfizer, and Santen. Portions of this study were presented in poster form at the annual meeting of the Association for Research in Vision and Ophthalmology, Fort Lauderdale, FL, May 1-5, 2011. The other authors report no conflicts of interest in this work.

\section{References}

1. Denton M, Kerr KG. Microbiological and clinical aspects of infection associated with Stenotrophomonas maltophilia. Clin Microbiol Rev. 1998;11:57-80.

2. Penland RL, Wilhelmus KR. Stenotrophomonas maltophilia ocular infections. Arch Ophthalmol. 1996;114:433-436.

3. Cho BJ, Lee GJ, Ha SY, Seo YH, Tchah H. Co-infection of the human cornea with Stenotrophomonas maltophilia and Aspergillus fumigatus. Cornea. 2002;21:628-631.

4. Rudolph T, Welinder-Olsson C, Lind-Brandberg L, Stenevi U. 16S rDNA PCR analysis of infectious keratitis: a case series. Acta Ophthalmol Scand. 2004;82:463-467.

5. Mauger TF, Craig E. Combined acanthamoeba and Stenotrophomonas maltophilia keratitis treated with a conjunctival flap followed by penetrating keratoplasty. Cornea. 2006;25:631-633.

6. Kim JH, Shin HH, Song JS, Kim HM. Infectious keratitis caused by Stenotrophomonas maltophilia and yeast simultaneously. Cornea. 2006;25:1234-1236.

7. Mauger TF, Kuennen RA, Smith RH, Sawyer W. Acanthamoeba and Stenotrophomonas maltophilia keratitis with fungal keratitis in the contralateral eye. Clin Ophthalmol. 2010;21:1207-1209.

8. Holifield K, Lazzaro DR. Case report: spontaneous Stenotrophomonas maltophilia keratitis in a diabetic patient. Eye Contact Lens. 2011;37: 326-327.

9. Son SW, Kim HJ, Seo JW. A case of Stenotrophomonas maltophilia keratitis effectively treated with moxifloxacin. Korean J Ophthalmol. 2011;25:349-351.

10. Chen YF, Chung PC, Hsiao CH. Stenotrophomonas maltophilia keratitis and scleritis. Chang Gung Med J. 2005;28:142-150.
11. Ramos-Esteban JC, Jeng BH. Posttraumatic Stenotrophomonas maltophilia infectious scleritis. Cornea. 2008;27:232-235.

12. Lin HC, Ma DH, Chen YF, Yeh LK, Hsiao CH. Late-onset intrascleral dissemination of Stenotrophomonas maltophilia scleritis after pterygium excision. Cornea. 2011;30:712-715.

13. Wladis EJ. Dacryocystitis secondary to Stenotrophomonas maltophilia infection. Ophthal Plast Reconstr Surg. 2011;27:e116-e117.

14. Marx DP, Chang PT, Winthrop KL. Strenotrophomonas maltophiliarelated chronic dacryocystitis. Orbit. 2012;31:433-434.

15. Mahendradas P, Avadhani K, Anandula V, Shetty R. Unilateral conjunctival ulcer due to Stenotrophomonas maltophilia infection. Indian J Ophthalmol. 2012;60:134-136.

16. Liu DT, Lee VY, Chi-Lai L, Lam DS. Stenotrophomonas maltophilia and Mycobacterium chelonae coinfection of the extraocular scleral buckle explant. Ocul Immunol Inflamm. 2007;15:441-442.

17. Das T, Deshmukh HS, Mathai A, Reddy AK. Stenotrophomonas maltophilia endogenous endophthalmitis: clinical presentation, sensitivity spectrum and management. J Med Microbiol. 2009;58(Pt 6):837-838.

18. Patton N. Post-traumatic endophthalmitis caused by Xanthomonas maltophilia. Eye (Lond). 2001;15(Pt 6):801-802.

19. Lai TY, Kwok AK, Fung KS, Chan WM, Fan DS, Lam DS. Stenotrophomonas maltophilia endophthalmitis after penetrating injury by a wooden splinter. Eye (Lond). 2001;15(Pt 3):353-354.

20. Kherani F, Kherani A, Gehrs KM, Heilskov TW, Sutphin JE, Wagoner MD. Xanthomonas maltophilia endophthalmitis following penetrating corneal injury. Can J Ophthalmol. 2002;37:301-303.

21. Essex RW, Charles PG, Allen PJ. Three cases of post-traumatic endophthalmitis caused by unusual bacteria. Clin Experiment Ophthalmol. 2004;32:445-447.

22. Horster S, Bader L, Seybold U, Eschler I, Riedel KG, Bogner JR. Stenotrophomonas maltophilia induced post-cataract-surgery endophthalmitis: outbreak investigation and clinical courses of 26 patients. Infection. 2009;37:117-122.

23. Akçakaya AA, Sargin F, Erbil HH, et al. A cluster of acute-onset postoperative endophthalmitis over a 1-month period: investigation of an outbreak caused by uncommon species. Br J Ophthalmol. 2011;95:481-484.

24. Chen S, Stroh EM, Wald K, Jalkh A. Xanthomonas maltophilia endophthalmitis after implantation of sustained-release gancyclovir. Am J Ophthalmol. 1992;114:772-773.

25. Kaiser GM, Tso PC, Morris R, McCurdy D. Xanthomonas maltophilia endophthalmitis after cataract extraction. Am J Ophthalmol. 1997;123: $410-411$.

26. Chaudhry NA, Flynn HW Jr, Smiddy WE, Miller D. Xanthomonas maltophilia endophthalmitis after cataract surgery. Arch Ophthalmol. 2000;118:572-575.

27. Horio N, Horiguchi M, Murakami K, Yamamoto E, Miyake Y. Stenotrophomonas maltophilia endophthalmitis after intraocular lens implantation. Graefes Arch Clin Exp Ophthalmol. 2000;238:299-301.

28. Benian O, Alimgil L, Erda N. Two cases of Stenotrophomonas maltophilia endophthalmitis. Ophthalmic Surg Lasers. 2002;33:253-256.

29. Karakurt A, Abdik O, Sengün A, et al Stenotrophomonas maltophilia endophthalmitis after cataract extraction. Ocul Immunol Inflamm. 2006; 14:41-46.

30. Jamrozy-Witkowska AM, Grzybowski A, Grabska-Liberek I, Pawlik-Sobecka LA, Krzyżanowska-Berkowska PP. Co-existence of Staphylococcus spp and Stenotrophomonas maltophilia infection in persistent endophthalmitis after cataract surgery. Clin Exp Optom. 2010;93:473-475.

31. Chen KJ, Wang NK, Sun MH, et al. Endophthalmitis caused by Stenotrophomonas maltophilia. Ophthalmic Surg Lasers Imaging. 2010;41:e555-e561.

32. Endophthalmitis Vitrectomy Study Group. Results of the Endophthalmitis Vitrectomy Study. Arch Ophthalmol. 1995;113:1479-1496.

33. Lalwani GA, Flynn HW Jr, Scott IU, et al. Acute-onset endophthalmitis after clear corneal cataract surgery (1996-2005) : clinical features, causative organisms, and visual acuity outcomes. Ophthalmology. $2008 ; 115: 473-476$. 
Clinical Ophthalmology

\section{Publish your work in this journal}

Clinical Ophthalmology is an international, peer-reviewed journal covering all subspecialties within ophthalmology. Key topics include: Optometry; Visual science; Pharmacology and drug therapy in eye diseases; Basic Sciences; Primary and Secondary eye care; Patien Safety and Quality of Care Improvements. This journal is indexed on

PubMed Central and CAS, and is the official journal of The Society of Clinical Ophthalmology (SCO). The manuscript management system is completely online and includes a very quick and fair peer-review system, which is all easy to use. Visit http://www.dovepress.com/ testimonials.php to read real quotes from published authors. 